\title{
Is this the right MOMENTUM? - evidence from a HeartMate 3 randomized trial
}

\author{
Silvia Mariani, Anamika Chatterjee, Jasmin S. Hanke, Katharina Homann, Günes Dogan, Axel Haverich, \\ Jan D. Schmitto \\ Department of Cardiothoracic, Transplantation and Vascular Surgery, Hannover Medical School, Hannover, Germany \\ Correspondence to: Prof. Dr. Jan D. Schmitto, MD, PhD, MBA, FCCP, FRCS (Glasg.), FACS. Department of Cardiothoracic, Transplant and Vascular \\ Surgery, Medizinische Hochschule Hannover, Carl-Neuberg-Str. 1, 30625 Hannover, Germany. Email: Schmitto.Jan@mh-hannover.de. \\ Provenance: This is an invited article commissioned by the Academic Editor Zhizhou Yang (Washington University School of Medicine in St. Louis, \\ St. Louis, MO, USA). \\ Comment on: Mehra MR, Uriel N, Naka Y, et al. A Fully Magnetically Levitated Left Ventricular Assist Device - Final Report. N Engl J Med \\ 2019;380:1618-27.
}

Submitted Nov 14, 2019. Accepted for publication Nov 22, 2019.

doi: $10.21037 /$ jtd.2019.11.60

View this article at: http://dx.doi.org/10.21037/jtd.2019.11.60

Left ventricular assist devices (LVADs) are widely used in selected patients suffering from end-stage heart failure (HF). However, their large-scale use has been limited in the past by their adverse events rate. With the development of the third LVAD generation characterized by centrifugal pumps, the role and application of LVADs for treating HF patients has globally increased with improving results, better quality of life and survival. This led to a growing need for scientific evidence supporting the large-scale application of this therapy.

Part of this success is due also to the newest generation of LVADs, especially the HeartMate 3 (Abbott, Inc, IL, USA), a centrifugal pump represented in Figure 1 and implanted for the first time worldwide in a patient by Dr. Schmitto and his team at Hannover Medical School, Hannover, Germany (1). Positive results of this first implant were subsequently followed by a multicenter European trial which brought HeartMate3 (HM3) to its CE mark approval in 2015 (2). A subsequent post-market multinational registry recorded HM3 results in a real-life scenario of 26 cardiac surgery centers (3). In parallel, Mehra et al. completed a multicenter randomized FDA trial comparing HM3 with HeartMate II (HMII) in patients with advanced HF (4). The MOMENTUM 3 trial, or Multi-center Study of MagLev Technology in patients undergoing MCS Therapy With HeartMate 3, enrolled 1,028 patients who were randomized to implantation of an HM3 or HMII from September 2014 to August 2016 (4). All patients were monitored for two years and the study demonstrated that a larger percentage of patients in the HM3 group compared to the axial-flow pump group remained alive and free of disabling stroke or reoperation to replace or remove a malfunctioning device at 2 years $(76.9 \%$ vs. $64.8 \%)$ (4). This study marked the definitive superiority of the third LVAD generation over HMII determining the end of axial-flow pumps era. When analyzing the actuarial overall survival, $79 \%$ of HM3 patients and $76.7 \%$ of HMII patients were alive at 2 years. This result was already described in the CE Mark trial which observed a 2 -year survival of $74 \%$ in a mixed population including both bridge to transplant and destination therapy patients. Both the CE Mark trial and the MOMENTUM 3 trial are in line with results presented by the INTERMACS report demonstrating a 24-month survival of $76 \%$ for centrifugal pumps and $74 \%$ for axialflow pumps $(4,5)$.

If the survival rates described in the MOMENTUM 3 trial are in line with previous studies, more attention should be placed on other components of the primary endpoint investigated by Mehra et al. (4). Indeed, the HM3 superiority is mainly based on its lower rate of disabling strokes and reoperation to replace or remove a malfunctioning device. Pump replacement at 2 years occurred in $2.3 \%$ of patients who received a centrifugal-flow pump and in $11.3 \%$ of patients receiving HMII (4). The axial-flow pumps required 


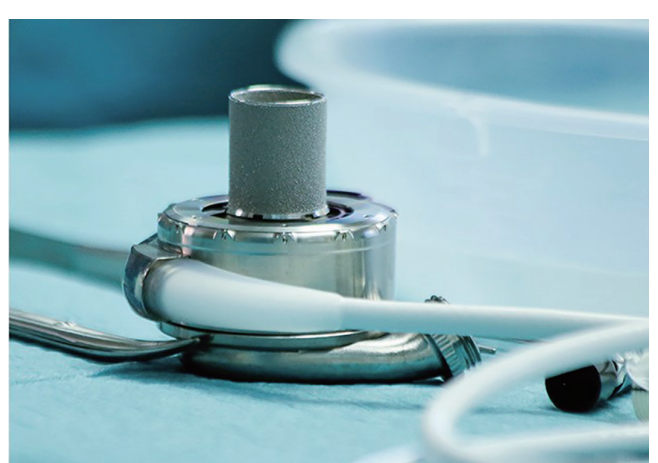

Figure 1 HeartMate 3 (Abbott, Inc, IL, USA) pump during the assembly procedure before implantation.

replacement for suspected thrombosis in 44 cases while this happened only in 3 patients in the HM3 group (4). Even better results were described in the CE Mark trial, the ELEVATE registry and in first clinical experiences with HM3 where no pump thrombosis were observed in the whole population $(2,3,6)$. This is explained by HM3 design which avoids any mechanical contact point and by its large sintered surfaces intended to ensure hemocompatibility of blood contacting components. As consequence of this HM3 lower thrombogenicity, lactate dehydrogenase (LDH) levels were much lower in the centrifugal-flow pump group (at 24 months: $252 \pm 95 \mathrm{U} / \mathrm{L}$ ) than in the axial-flow pump group (at 24 months: $344 \pm 190 \mathrm{U} / \mathrm{L}$ ) in the MOMENTUM 3 trial (4).

In addition to a reduced rate of pump thrombosis in the MOMENTUM 3 trial (relative risk: 0.08; 95\% CI: 0.04-0.16), HM3 was associated with a lower risk of either ischemic or hemorrhagic strokes of any severity (relative risk: 0.42 ; 95\% CI:0.30-0.57) (4). While all previously discussed data are comparable among trials, the rate of all strokes for HM3 is lower in MOMENTUM 3 trial compared to the CE Mark trial $(2,4)$. Similarly, HVAD (HeartWare $^{\circledR}$, Medtronic, MN, USA) demonstrated a stroke rate of 0.07 EPPY in the ReVOLVE study (7) and 0.10 EPPY in the recent LATERAL trial $(8,9)$ while it had a stroke rate of 0.23 EPPY in the ENDURANCE Supplemental trial $(9,10)$. For both HM3 and HVAD, the best neurologic outcomes were achieved in the most recent studies. This might be explained with differences among baseline patients' characteristics and clinical management (9). A more detailed analysis of stroke events in the long-term cohort of the MOMENTUM 3 study showed that strokes occurred at a median time of 131 days after implant (11). Colombo et al. did not observe differences between HM3 and HM II in terms of stroke rates from implant to 180 days of follow-up. However, when analyzing stroke incidence in the long-term period (181-730 days of follow-up), this incidence was 3.3 times lower for the HM3 group (relative risk: 0.23 ; $95 \%$ CI: 0.08-0.63). Therefore, it can be speculated that the protective effects of HM3 in terms of neurological events are much more evident with time rather than in the shortterm follow-up. Interestingly, previous studies including patients who received a different pump (e.g., HVAD) identified systemic hypertension as a predictor of stroke and mean arterial pressure lower than $90 \mathrm{mmHg}$ as protective factor (12). Differently, the MOMENTUM 3 study did not demonstrate any conclusive associations between blood pressure, anticoagulation, and antiplatelet therapy with respect to occurrence of strokes by either device (11).

When discussing the topic of hemocompatibility, strokes and pump thrombosis should be analyzed in relation to bleeding events. As a matter of fact, the delicate balance between thrombosis and bleeding is one of the main concerns in the management of patients receiving mechanical circulatory support (MCS) since the strategies to lower one of these drawbacks might directly influence the other side of this delicate equilibrium. In MOMENTUM 3 study (4), HM3 was associated with lower rates of bleeding compared to HMII (relative risk: 0.64; 95\% CI: 0.57-0.72) and similar rates were already described by the CE Mark trial (Table 1) (2). These results can again be explained with some pump features such as a frictionless rotor and wide blood filled gaps between the rotor and the housing able to reduce shear stress and blood damage. Moreover, the HMII group in the MOMENTUM 3 trial underwent a more aggressive antiplatelet therapy in the long-term followup probably as a consequence of the higher thrombotic burden. Indeed, at 2 years of follow-up the percentage of patients receiving more than one anti-platelet medication was double in the HMII group (12.9\%) than in the HM3 group (6.6\%). A previous analysis of MOMENTUM 3 hemocompatibility outcomes suggested the use of an aggregate of hemocompatibility-related clinical adverse events (HRAEs). Uriel et al. applied a HRAEs score to the first cohort of patients enrolled in the MOMENTUM 3 trial and followed-up for 6 months (13). This study led again to the superiority of HM3 compared to HMII predicting the results of the MOMENTUM 3 final report. However, a significant residual risk of bleeding persists in the HM3 cohort and this should not be underestimated in 
Table 1 Adverse event rates expressed as events/patient-year. Data from MOMENTUM 3 trial refer to the per protocol analysis

\begin{tabular}{|c|c|c|c|}
\hline Adverse event & $\begin{array}{c}\text { CE Mark } \\
\text { trial }(2) \\
\text { HM3 }(n=50)\end{array}$ & $\begin{array}{c}\text { MOMENTUM } 3 \\
\text { trial }(4) \\
\text { HM3 }(n=515)\end{array}$ & $\begin{array}{c}\text { MOMENTUM } 3 \\
\text { trial (4) HM II } \\
(n=505)\end{array}$ \\
\hline Pump thrombosis & 0.00 & 0.01 & 0.12 \\
\hline \multicolumn{4}{|l|}{ Stroke } \\
\hline Any stroke & 0.15 & 0.08 & 0.18 \\
\hline Hemorrhagic stroke & 0.05 & 0.03 & 0.07 \\
\hline Ischemic stroke & 0.10 & 0.04 & 0.11 \\
\hline \multicolumn{4}{|l|}{ Bleeding } \\
\hline Any bleeding & 0.67 & 0.61 & 0.95 \\
\hline Requiring surgery & 0.14 & 0.08 & 0.14 \\
\hline \multicolumn{4}{|l|}{ Infections } \\
\hline Any infection & 0.61 & 0.82 & 0.82 \\
\hline Sepsis & 0.14 & 0.13 & 0.13 \\
\hline Driveline infection & 0.22 & 0.23 & 0.22 \\
\hline \multicolumn{4}{|l|}{ Right heart failure } \\
\hline $\begin{array}{l}\text { Any right heart } \\
\text { failure }\end{array}$ & 0.09 & 0.27 & 0.23 \\
\hline Requiring RVAD & 0.03 & 0.03 & 0.03 \\
\hline
\end{tabular}

HM II, HeartMate II; HM3, HeartMate 3; RVAD, right ventricular assist device.

the real-world clinical life. The enthusiasm for low stroke rates in HM3 patients pushed the scientific community toward the investigation of low-intensity anti-coagulation regimens in patients receiving HM3 support (14). The MAGENTUM 1 study tested the feasibility of a lowintensity anti-coagulation regimen in 15 patients (14). After standard warfarin anti-coagulation with international normalized ratio (INR) between 2.0 and 3.0 and aspirin for 6 weeks post-implant, patients were treated with a lower INR target range of 1.5 to 1.9 (14). No episodes of clinically relevant hemolysis were noted and only one patient experience suspected gastrointestinal bleeding with drop in hemoglobin (14). The authors concluded that low-intensity anti-coagulation is feasible and is not associated with an increase in thromboembolic complications. However, the study cohort was extremely small and further larger studies are mandatory to prove this hypothesis. If the safety of this anti-coagulation approach will be proved, it will determine an important reduction in non-surgical bleeding and a massive improvement in patients' quality of life and outcomes.

In addition to hemocompatibility outcomes, the MOMENTUM 3 study recorded all sort of infections occurring in the analyzed population. As stated by the authors, infections occurred quite frequently with no significant differences between devices (4). While the incidence of overall infections was slightly lower in the CE Mark trial as shown in Table 1 (2), the rates of driveline infections and sepsis were comparable between the two studies (4). Despite the rapid technological evolution of pump design, driveline-related problems still represent a huge burden for patients. MOMENTUM 3 trial clearly demonstrated that no significant improvements have been made in terms of infections in the last two LVAD generations and the whole clinical community is looking with interest at the development of fully implantable devices. The use of transcutaneous energy transmission systems will definitely eliminate all driveline-related complications and, probably, lower the overall risk of infections.

After discussing nearly all major adverse events, the authors of the MOMENTUM 3 study focused their attention on a peculiar complication that has been described as typically related to HM3. Outflow graft twist has first been noticed in HM3 (15) and it is characterized by a rotation of the outflow graft leading to reduction or disruption of the pump flow. Such a complication requires surgery and it usually appears late after pump implantation. The MOMENTUM 3 trial reported 8 cases of outflow graft twist with an incidence of $1.6 \%$ (95\% CI: $0.7-3.0 \%$ ) (16). This incidence rate is double compared to the previously recorded value of $0.72 \%$ (95\% CI: $0.5-1.0 \%$ ) reported by the manufacturer in 2018 (16). Both these numbers should be kept in mind when performing follow-up visits in patients implanted with HM3 before 2018 . In case of persistent low-flows without other clinical explanations, a contrast enhanced CT-scan is advised to diagnose or exclude deformations of the outflow grafts resulting from graft torsions. In the MOMENTUM 3 cohort (16), outflow graft twist was observed at a median follow-up of 544 days (range, 347-688 days) and surgery was performed in all cases to correct the torsion or exchange the graft (62.5\%), exchange the pump $(25 \%)$ or perform urgent transplantation (12.5\%) (16). The authors reported a $1.6 \mathrm{~L} / \mathrm{min}$ median reduction in pump flow due to graft twist and an increase in $\mathrm{LDH}$ values in $50 \%$ of cases (16). After 2018, a surgical clip to fix the pump outflow graft to the connector was developed to 
prevent outflow-graft rotation. Further follow-up data are required to confirm the efficacy of this new clip used in the most recent $\mathrm{HM} 3$ implants.

While the MOMENTUM 3 trial reported a detailed picture of HM3-related outcomes, it should be kept in mind that all presented results were recorded in a highly selected population, as it is common in complex randomized trials. This method allows for control of selection bias but it excludes a wide segment of the typical LVAD population. An example is the exclusion of patients treated with ongoing MCS other than IABP. This means that all patients bridged to LVAD with extracorporeal membrane oxygenation or other temporary MCS were excluded. Moreover, all patients previously supported with other kind of LVADs were not considered in this study. In our experience, LVAD exchanges from HM II or HVAD to HM 3 with minimally invasive technique is feasible and it is an excellent opportunity to give patients access to the latest generation of assist device (17). Due to the higher complexity of surgery and postoperative management, patients undergoing biventricular VAD (Bi-VAD) support were excluded in the MOMENTUM 3 trial. However, literature already reports results from the first international multicenter experience with HM3 used as Bi-VAD (18). In this case series of 14 patients, 8 of them were on Bi-VAD support for 95-636 days, 7 of them were discharged at home and one was successfully transplanted. Additionally, HM3 showed a low incidence of thrombosis as well when used as right ventricular assist device (18). Two HM3 can also be implanted in the configuration of a total artificial heart after excision of both ventricles (18). Even if these HM3 applications are not considered in the MOMENTUM 3 trial, surgeons regularly dealing with LVAD implantation should be aware of these possibilities and future studies should further investigate their outcomes.

Another interesting aspect of MOMENTUM 3 results is the small number of patients who underwent explantation or deactivation. In the primary endpoint analysis, the authors considered two patients in the axial-flow pump group who underwent explantation or deactivation of the device for reasons other than myocardial recovery, as treatment failures (4). However, in the competing risk analysis they report that $1 \%$ of HM3 patients also received explantation or deactivation of the pump, presumably for myocardial recovery (not explicitly specified). No further details were provided on the HM3 explantation technique. Several explantation strategies for different LVADs have been described, including complete removal of all LVAD components followed by a ventriculoplasty or isolated pump removal and closure of the ventriculotomy with a patch. In our experience, we prefer to explant the pump and ligate the outflow graft, while leaving the inflow sewing ring in place. As with this technique a strategy to occlude the ventriculotomy is required, we designed a novel custommade apical ring plug which facilitates a minimally invasive implantation approach (19).

In summary, the MOMENTUM trial published by Dr. Mehra on behalf of all investigators of the FDA-study demonstrated the superiority of HM3 compared to HMII and accomplished one of the most relevant and valuable randomized trials in the field of LVADs. Results described in this study confirm what other authors already observed in the CE Mark trial (2), in the ELEVATE registry (3) and other real-world clinical experiences (6). While in many centers worldwide HM3 replaced HMII in the clinical practice a while ago, the MOMENTUM 3 trial provides the scientific evidence of what was already clear in the clinical practice. A deep analysis of all MOMENTUM 3 data depicts a picture of a LVAD field which is rapidly improving in terms of numbers of implants, safety and efficacy but is still not free from adverse events and complications. Bleeding, embolic events, stroke and infections still affect outcomes after device implantation. Moreover, studies such as the MOMENTUM 3 trial are not able to capture and highlight the need for improvements in several ancillary aspects of LVAD technology such as pulsatility or adaption to patient's physical activity. Further technological developments and high-quality basic and clinical research in the direction of better biocompatible and physiological LVAD device are, therefore, urgently needed. Given the scientific evidences and the everyday clinical experience, we can conclude that the MOMENTUM 3 trial depicts a good picture of the current LVAD field with all its lights and shadows. However, despite its good results, it should not be interpreted as a satisfying destination but rather like a single momentum able to push the LVAD field forward.

\section{Acknowledgments}

None.

\section{Footnote}

Conflicts of Interest: JD Schmitto and G Dogan are consultants for Medtronic, MN, USA and Abbott, IL, USA. 
The other authors have no conflicts of interest to declare.

Ethical Statement: The authors are accountable for all aspects of the work in ensuring that questions related to the accuracy or integrity of any part of the work are appropriately investigated and resolved.

\section{References}

1. Schmitto JD, Hanke JS, Rojas SV, et al. First implantation in man of a new magnetically levitated left ventricular assist device (HeartMate III). J Heart Lung Transplant 2015;34:858-60.

2. Schmitto JD, Pya Y, Zimpfer D, et al. Long-term evaluation of a fully magnetically levitated circulatory support device for advanced heart failure-two-year results from the HeartMate 3 CE Mark Study. Eur J Heart Fail 2019;21:90-7.

3. Garbade J, Gustafsson F, Shaw S, et al. Postmarket Experience With HeartMate 3 Left Ventricular Assist Device: 30-Day Outcomes From the ELEVATE Registry. Ann Thorac Surg 2019;107:33-9.

4. Mehra MR, Uriel N, Naka Y, et al. A Fully Magnetically Levitated Left Ventricular Assist Device - Final Report. N Engl J Med 2019;380:1618-27.

5. Kormos RL, Cowger J, Pagani FD, et al. The Society of Thoracic Surgeons Intermacs database annual report: Evolving indications, outcomes, and scientific partnerships. J Heart Lung Transplant 2019;38:114-26.

6. Hanke JS, Dogan G, Rojas SV, et al. First experiences with HeartMate 3 follow-up and adverse events. J Thorac Cardiovasc Surg 2017;154:173-8.

7. Schmitto JD, Zimpfer D, Fiane AE, et al. Long-term support of patients receiving a left ventricular assist device for advanced heart failure: a follow-up analysis of the Registry to Evaluate the HeartWare Left Ventricular Assist System. Eur J Cardiothorac Surg 2016;50:834-8.

8. McGee E Jr, Danter M, Strueber M, et al. Evaluation of a lateral thoracotomy implant approach for a centrifugalflow left ventricular assist device: The LATERAL clinical trial. J Heart Lung Transplant 2019;38:344-51.

Cite this article as: Mariani S, Chatterjee A, Hanke JS, Homann K, Dogan G, Haverich A, Schmitto JD. Is this the right MOMENTUM? - evidence from a HeartMate 3 randomized trial. J Thorac Dis 2019;11(12):5626-5630. doi: 10.21037/jtd.2019.11.60
9. Li S, Beckman JA, Cheng R, et al. Comparison of Neurologic Event Rates Among HeartMate II, HeartMate 3, and HVAD. ASAIO J 2019. [Epub ahead of print].

10. Milano CA, Rogers JG, Tatooles AJ, et al. HVAD: The ENDURANCE Supplemental Trial. JACC Heart Fail 2018;6:792-802.

11. Colombo PC, Mehra MR, Goldstein DJ, et al. Comprehensive Analysis of Stroke in the Long-Term Cohort of the MOMENTUM 3 Study. Circulation 2019;139:155-68.

12. Teuteberg JJ, Slaughter MS, Rogers JG, et al. The HVAD Left Ventricular Assist Device: Risk Factors for Neurological Events and Risk Mitigation Strategies. JACC Heart Fail 2015;3:818-28.

13. Uriel N, Colombo PC, Cleveland JC, et al. Hemocompatibility-Related Outcomes in the MOMENTUM 3 Trial at 6 Months: A Randomized Controlled Study of a Fully Magnetically Levitated Pump in Advanced Heart Failure. Circulation 2017;135:2003-12.

14. Netuka I, Ivák P, Tučanová Z, et al. Evaluation of lowintensity anti-coagulation with a fully magnetically levitated centrifugal-flow circulatory pump-the MAGENTUM 1 study. J Heart Lung Transplant 2018;37:579-86.

15. Grüger T, Kaufmann F, Dreysse S, et al. Late post-pump blood flow obstruction in a novel left ventricular assist device: The unusual case of a twisted outflow graft. J Thorac Cardiovasc Surg 2018;155:e33-5.

16. Mehra MR, Salerno C, Naka Y, et al. A tale of the twist in the outflow graft: An analysis from the MOMENTUM 3 trial. J Heart Lung Transplant 2018;37:1281-4.

17. Hanke JS, Rojas SV, Dogan G, et al. First series of left ventricular assist device exchanges to HeartMate 3. Eur J Cardiothorac Surg 2017;51:887-92.

18. Lavee J, Mulzer J, Krabatsch T, et al. An international multicenter experience of biventricular support with HeartMate 3 ventricular assist systems. J Heart Lung Transplant 2018;37:1399-402.

19. Ricklefs M, Deodhar C, Chatterjee A, et al. A new tool for an explantation strategy of HeartMate $3^{\mathrm{TM}}$ left ventricular assist device. J Thorac Dis 2018;10:S1825-8. 